\title{
Giardia fatty acyl-CoA synthetases as potential drug targets
}

\section{Fengguang Guo ${ }^{1}$, Guadalupe Ortega-Pierres ${ }^{2 *}$, Raúl Argüello-Garcia ${ }^{2}$, Haili Zhang ${ }^{1}$ and Guan Zhu'*}

' Department of Veterinary Pathobiology, College of Veterinary Medicine and Biomedical Sciences, Texas A\&M University, College Station, Texas, USA, ${ }^{2}$ Department of Genetics and Molecular Biology, Center for Research and Advanced Studies of the National Polytechnic Institute, Mexico City, Mexico

Edited by:

Anjan Debnath,

University of California, San Diego,

USA

Reviewed by:

Dinesh Sriramulu,

Shres Consultancy, India

Larissa M. Podust,

University of California, San Diego,

USA

*Correspondence:

Guadalupe Ortega-Pierres,

Department of Genetics

and Molecular Biology, Center for Research and Advanced Studies of the National Polytechnic Institute,

Avenue Instituto Politécnico Nacional 2508, San Pedro Zacatenco,

07360 Mexico City, Mexico

gortega@cinvestav.mx; Guan Zhu,

Department of Veterinary Pathobiology, College of Veterinary Medicine and Biomedical Sciences,

Texas A\&M University, College

Station, Texas 77843-4467, USA

gzhu@cvm.tamu.edu

Specialty section:

This article was submitted to

Antimicrobials, Resistance and Chemotherapy,

a section of the journal

Frontiers in Microbiology

Received: 22 March 2015

Accepted: 08 July 2015

Published: 22 July 2015

Citation:

Guo F, Ortega-Pierres G,

Argüello-García $R$, Zhang $H$

and Zhu G (2015) Giardia fatty acyl-CoA synthetases as potential drug targets.

Front. Microbiol. 6:753. doi: 10.3389/fmicb.2015.00753
Giardiasis caused by Giardia intestinalis (syn. G. lamblia, G. duodenalis) is one of the leading causes of diarrheal parasitic diseases worldwide. Although limited drugs to treat giardiasis are available, there are concerns regarding toxicity in some patients and the emerging drug resistance. By data-mining genome sequences, we observed that $G$. intestinalis is incapable of synthesizing fatty acids (FA) de novo. However, this parasite has five long-chain fatty acyl-CoA synthetases (GiACS1 to GiACS5) to activate FA scavenged from the host. ACS is an essential enzyme because FA need to be activated to form acyl-CoA thioesters before they can enter subsequent metabolism. In the present study, we performed experiments to explore whether some GiACS enzymes could serve as drug targets in Giardia. Based on the high-throughput datasets and protein modeling analyses, we initially studied the GiACS1 and GiACS2, because genes encoding these two enzymes were found to be more consistently expressed in varied parasite life cycle stages and when interacting with host cells based on previously reported transcriptome data. These two proteins were cloned and expressed as recombinant proteins. Biochemical analysis revealed that both had apparent substrate preference toward palmitic acid (C16:0) and myristic acid (C14:0), and allosteric or Michaelis-Menten kinetics on palmitic acid or ATP. The ACS inhibitor triacsin C inhibited the activity of both enzymes $\left(\mathrm{IC}_{50}=1.56 \mu \mathrm{M}, K_{\mathrm{i}}=0.18 \mu \mathrm{M}\right.$ for GiACS1, and $\mathrm{IC}_{50}$ $=2.28 \mu \mathrm{M}, K_{\mathrm{i}}=0.23 \mu \mathrm{M}$ for GiACS2, respectively) and the growth of $\mathrm{G}$. intestinalis in vitro $\left(\mathrm{IC}_{50}=0.8 \mu \mathrm{M}\right)$. As expected from giardial evolutionary characteristics, both GiACSs displayed differences in overall folding structure as compared with their human counterparts. These observations support the notion that some of the GiACS enzymes may be explored as drug targets in this parasite.

Keywords: Giardia intestinalis, fatty acyl-CoA synthetases, drug target, protein modeling, triacsin-C

\section{Introduction}

Giardia intestinalis (syn. G. lamblia, G. duodenalis) is one of the major causative agents of diarrheal diseases in humans around the world. In the U.S. alone, there are estimated 1.2-2 million (but up to 8 million) cases per year, resulting in annual costs of $>\$ 30$ million USD (Hlavsa et al., 2005; Yoder et al., 2007, 2010, 2012). The recently reported infection rates range from $0.4-7.6 \%$ in developed countries, and 0.9-40\% in developing countries (Plutzer et al., 2010; Feng and Xiao, 2011). Because Giardia cysts exhibit moderate resistance to the chlorine used in treating water for drinking and 
recreational purposes, it is also one of the most common waterborne pathogens and is listed as a Category B priority pathogen in the NIH/CDC Biodefense program ${ }^{1}$. In addition to water-borne and food-borne transmissions, $G$. intestinalis is also a zoonotic pathogen capable of transmitting infections between animals and humans. Drugs to treat giardiasis are available, but the choices are limited (e.g., metronidazole, tinidazole, albendazole, and nitazoxanide) (Busatti et al., 2009; Tian et al., 2010; TejmanYarden and Eckmann, 2011; Granados et al., 2012). These drugs are also not $100 \%$ effective and may be unsuitable for some patients due to toxicity. Drug resistance is also an emerging problem (Ali and Nozaki, 2007; Lalle, 2010; Tian et al., 2010). Therefore, new or alternative drugs are needed, particularly if massive infection occurs under natural or man-made conditions.

Giardia species are anaerobic protozoa evolutionarily branched early at the base of eukaryotes (Morrison et al., 2007). The genome of $G$. intestinalis has been sequenced and reported in 2007, which revealed that this parasite has none or limited ability to synthesize most nutrients de novo, such as amino acids, nucleosides, and fatty acids (FA) (Morrison et al., 2007). In FA metabolism, Giardia lacks both types I and II synthetic pathways, and thus relies on scavenging FAs from hosts. This notion is also supported by earlier biochemical analysis on this parasite (Jarroll et al., 1981; Beach et al., 1990; Das et al., 2002; Lee et al., 2007). This anaerobic protozoan retains limited fatty acyl extension ability by possessing one or more elongating (ELO) genes. Congruent with its anaerobic life style, Giardia also lacks enzymes for FA degradation and $\beta$-oxidation. FA scavenged from hosts are first activated by acyl-CoA synthetase (ACS, aka. FA-CoA ligase, ACL) to form fatty acyl-CoA (FA-CoA) thioesters before they can enter to subsequent metabolic pathways, such as FA elongation and synthesis of lipids and biomembranes (Figures 1A,B). Therefore, targeting ACS may block the entire FA metabolism, thus killing the parasite.

In this study, we cloned and expressed two of the five ACSs from G. intestinalis (named as GiACS1 and GiACS2) as maltosebinding protein (MBP)-fusion proteins, and characterized their

${ }^{1}$ http://www.niaid.nih.gov/topics/BiodefenseRelated/Biodefense/Pages/CatA. aspx substrate preference and enzyme kinetic features. We also showed that the ACS inhibitor triacsin C could not only inhibit the activity of GiACS1 and GiACS2, but also display efficacy against the growth of $G$. intestinalis in vitro at micromolar levels.

\section{Materials and Methods}

\section{Data-mining the G. intestinalis ACS Genes and their Expression Profiles}

To ensure the full recovery of ACS genes from the G. intestinalis genomes, we searched the Giardia $\mathrm{DB}^{2}$ and the Giardia reference genomes at the National Center for Biotechnology Information ${ }^{3}$ (NCBI) with relevant keywords and by BLAST searches using known long-chain fatty acyl (LCFA)-CoA protein sequences as queries. The identities of GiACS proteins were further confirmed by BLAST searches for their orthologs and signature domains at the NCBI Conserved Domain Database ${ }^{4}$ (CDD). This strategy identified five G. intestinalis ACS (GiACS) orthologs and three related genes that are summarized in Table $\mathbf{1}$.

By taking advantage of already published and publically available transcriptome datasets at the GiardiaDB, we also datamined the expression levels and fold changes of the five GiACS genes to evaluate their importance and potential differential roles in various parasite stages. These included their transcript levels in trophozoites and cysts, as well as during the encystation, excystation, and interactions with host cells that were determined by serial analysis of gene expression (SAGE), microarray analysis, and RNA-seq using the Illumina HiSeq2000 platform (Palm et al., 2005; Morf et al., 2010; Ringqvist et al., 2011; Franzen et al., 2013). Expression data of individual GiACS genes were extracted from corresponding datasets in GiardiaDB and values were plotted for comparison.

\section{Protein Modeling}

Structure homology modeling was performed on GiACS1 and GiACS2 (853 and 693 aa, respectively) using the I-Tasser

\footnotetext{
${ }^{2}$ http://www.giardiadb.org

${ }^{3}$ http://www.ncbi.nlm.nih.gov/

${ }^{4}$ http://www.ncbi.nlm.nih.gov/cdd/
}

TABLE 1 | Fatty acyl-CoA (FA-COA) synthetase (ACS) orthologs and related genes identified from the Giardia intestinalis genome and their top hits at the NCBI conserved domain (CDD) database.

\begin{tabular}{|c|c|c|c|c|c|c|c|}
\hline $\begin{array}{l}\text { Gene } \\
\text { Name }\end{array}$ & $\begin{array}{l}\text { GenBank } \\
\text { Accession No. }\end{array}$ & $\begin{array}{l}\text { GiardiaDB Gene } \\
\text { ID }\end{array}$ & GiardiaDB description & $\begin{array}{l}\text { Protein } \\
\text { size }\end{array}$ & CDD top hit & $\begin{array}{l}\text { CDD } \\
\text { accession }\end{array}$ & $\begin{array}{l}E \text {-value to } \\
\text { CDD top hit }\end{array}$ \\
\hline GiACS1 & XP_001705891 & GL50803_9062 & $\begin{array}{l}\text { Long-chain fatty acid (FA) } \\
\text { CoA ligase } 5\end{array}$ & 853 aа & LC-FACS_euk & cd05927 & $8.73 E-120$ \\
\hline GiACS2 & XP_001706424 & GL50803_15063 & Long-chain FA CoA ligase 5 & 693 aа & LC-FACS_euk & cd05927 & $1.92 E-157$ \\
\hline GiACS3 & XP_001705009 & GL50803_21118 & Long-chain FA CoA ligase 5 & 765 aa & LC-FACS_euk & cd05927 & $2.72 \mathrm{E}-117$ \\
\hline GiACS4 & XP_001708520 & GL50803_30476 & Long-chain FA CoA ligase 4 & 804 aа & LC-FACS_euk & cd05927 & 8.47E-163 \\
\hline GiACS5 & XP_001709411 & GL50803_113892 & $\begin{array}{l}\text { Long-chain FA CoA ligase, } \\
\text { putative }\end{array}$ & 758 aа & LC-FACS_euk & cd05927 & 5.37E-117 \\
\hline Unnamed & XP_001707853 & GL50803_17170 & Long-chain FA CoA ligase 5 & 1,523 aa & VL_LC_FACS_like & cd05907 & $6.85 E-19$ \\
\hline Unnamed & XP_001710279 & GL50803_86511 & Acyl-CoA synthetase (ACS) & 970 aa & ATP-grasp_5 & pfam13549 & 4.05E-38 \\
\hline Unnamed & XP_001709605 & GL50803_16667 & ACS & 905 aa & ATP-grasp_5 & pfam13549 & 1.16E-38 \\
\hline
\end{tabular}


(Iterative Threading ASSEmbly Refinement) webserver at http:// zhanglab.ccmb.med.umich.edu/I-TASSER/ (Zhang, 2008; Roy et al., 2010). This platform is the most widely used system to build structural protein models from query sequences using the solved crystal structures contained at the RCSB Protein Data Bank (PDB) as templates. The quality of the model is given by a C-score (range -5 to 2 ), which is an index that considers TM and RMSD scores and allows for the ranking of the degrees of similarity between query and template protein structures. The C-score is used in combination with the TM score (range 0-1) to obtain the best model (Yang et al., 2015). The root-mean-square-deviation (RMSD) score indicates a measure of the differences (in $\AA$ ) between values predicted by retrieved models and the values actually observed in PDB templates. The quality of protein models obtained was further visualized and tested by Ramachandran plots in the Discovery Studio v4.1 client (Accelrys ${ }^{\mathrm{TM}}$ ) software. To compare the overall folding of two given protein models, the PDB files generated by I-Tasser platform were submitted to the TM-Align platform ${ }^{5}$ that retrieves the RMSD and TM scores for the structural alignment of the proteins studied. According to PDB statistics, TM-scores below 0.2 corresponds to randomly chosen unrelated proteins, whereas a score higher than 0.5 match generally the same fold.

\section{Expression of Recombinant GiACS Proteins}

From identified GiACS genes, we chose to first clone and express two genes for potential functional analysis (i.e., GiACS1 and GiACS2, corresponding to the G. intestinalis WB strain Gene ID numbers GL50803_9062 and GL50803_15063, or GenBank accession numbers XP_001705891 and XP_001706424, respectively) (Table 1 ). Genomic DNA was isolated from the WB strain of $G$. intestinalis (ATCC \# 30957) using Qiagen DNeasy Blood \& Tissue Kit using protocol recommended for cultured cells. For biochemical analysis, the entire intron less open reading frames (ORFs) of GiACS1 and GiACS2 genes were amplified from the genome DNA by PCR using high-fidelity Pfu Turbo HotStart DNA polymerase (Agilent Technologies, Los Angeles, CA, USA). Linker sequences containing Bam $\mathrm{HI}$ and HindIII restriction sites were added to the sense and antisense primers, respectively (Table 2 ). The PCR products were digested by BamHI and HindIII, and ligated into the linearized pMAL-c2E vector. The ligated plasmids were transferred into the Rosetta 2 strain of Escherichia coli competent cells (Novagen) and cultured

${ }^{5}$ http://zhanglab.ccmb.med.umich.edu/TM-align/

TABLE 2 | Primers used in the cloning of GiACS1 and GiACS2 genes.

\begin{tabular}{llll}
\hline $\begin{array}{l}\text { Gene } \\
\text { Name }\end{array}$ & Orientation & Linker & Sequence $\left(\mathbf{5}^{\prime}-\mathbf{3}^{\prime} \mathbf{1}^{\mathbf{1}}\right.$ \\
\hline GiACS1 & Forward & BamHI & ctggatcCATGATCTTCCATTCTAAAAC \\
GiACS1 & Reverse & HindIII & gcaagcttCTCTCCTTATCAACCATGGCTTC \\
GiACS2 & Forward & BamHI & ctggatcCATGTCGGATTCATCTGCC \\
GiACS2 & Reverse & Sall & gcgtcgacCTTACTAGATGGTCTAGA \\
\hline
\end{tabular}

${ }^{1}$ Lower cases indicate added linker sequences. in LB agar plates containing $100 \mu \mathrm{g} / \mathrm{mL}$ ampicillin, from which plasmids were isolated from individual colonies by E.Z.N.A. plasmid DNA miniprep kit (Omega Bio-Tek, Atlanta, GA, USA) and sequenced by Sanger sequencing technique at the Texas A\&M University Gene Technologies Laboratory ${ }^{6}$ to confirm their identity and sequence accuracy.

The expression of MBP-fusion proteins was carried out as described (Guo and Zhu, 2012; Guo et al., 2014; Yu et al., 2014). Briefly, engineered plasmids were transferred into the Rosetta 2 strain of $E$. coli to grow colonies in LB agar plates containing $100 \mu \mathrm{g} / \mathrm{mL}$ ampicillin and $34 \mu \mathrm{g} / \mathrm{mL}$ chloramphenicol, from which bacterial colonies (less than 1 week old) were individually transferred into $25 \mathrm{~mL} \mathrm{LB}$ broth containing $0.2 \%$ glucose and allowed to grow at $37^{\circ} \mathrm{C}$ overnight. The next day, bacterial broths were diluted by $1: 100$ with fresh medium and incubated at $37^{\circ} \mathrm{C}$ for $2 \mathrm{~h}$ or until $\mathrm{OD}_{495}$ reached to $\sim 0.5$, followed by the induction of expression by isopropyl $\beta$-D-1-thiogalactopyranoside (IPTG) $(0.3 \mathrm{mM})$ at $16^{\circ} \mathrm{C}$ overnight. Bacteria were collected by centrifugation $(6000 \times g, 10 \mathrm{~min})$ and lysed by sonication in Tris- $\mathrm{HCl}$ buffer ( $\mathrm{pH} 7.5$ ), from which the recombinant proteins were purified by amylose resin-based affinity chromatography according to the manufacturer's instructions (New England Biolabs). The quality and quantity of purified proteins were analyzed by SDS-PAGE and Bradford assay using BSA as the protein standard. Proteins were used immediately after the purification or stored at $-20^{\circ} \mathrm{C}$.

\section{Biochemical Assays}

The ACS activity was determined by a five, 5'-dithio-bis-(2nitrobenzoate) (DTNB) colorimetric assay for both GiACS1 and GiACS2. In the assay, free CoA in reduced form (CoA-SH) reacted with DTNB to form 5-thionitrobenzoic acid that was measured at $\mathrm{OD}_{412}$ (Bernson, 1976; Zhuravleva et al., 2012; Guo et al., 2014). Reactions were carried out in $200 \mu \mathrm{L}$ Tris- $\mathrm{HCl}$ buffer (0.1 mM, pH 8.0) containing $10 \mathrm{mM} \mathrm{KCl,} 50 \mu \mathrm{M}$ CoA, $500 \mu \mathrm{M}$ ATP, $10 \mathrm{mM} \mathrm{MgCl}$, and $100 \mu \mathrm{M}$ FA. The concentrations of substrates and cofactors might be varied for determining their dose-response curves and FA with carbon chains ranging from C2:0 to C30:0 were used for determining substrate preference. Reactions were started by the addition of $1 \mu \mathrm{g}$ of recombinant MBP-GiACS proteins, followed by incubation at $32^{\circ} \mathrm{C}$ for $10 \mathrm{~min}$, and then stopped by heating samples at $80^{\circ} \mathrm{C}$ for $5 \mathrm{~min}$. Following sample cooling to room temperature, $4 \mu \mathrm{L}$ of DTNB $(5 \mathrm{mM})$ was added into each reaction that was allowed for color development for $5 \mathrm{~min}$, followed by the measurement of $\mathrm{OD}_{412}$ values with a Multiskan Spectrum spectrophotometer (Thermo Scientific). Serial concentrations of $\mathrm{CoA}$ in the same reaction buffer were assayed and used as standard curves for calculating the amounts of $\mathrm{CoA}$ reduced in experimental samples. Enzyme kinetic parameters were determined using varied concentrations of palmitic acid (10-600 $\mu \mathrm{M})$ and ATP $(10-3000 \mu \mathrm{M})$, respectively.

To confirm the formation of palmitoyl-CoA catalyzed by GiACS1 and GiACS2, we performed a radioactive assay in which reactions were carried out with the use of ${ }^{3} \mathrm{H}$-palmitic acid $(25 \mu \mathrm{M})$ and other reagents as described above. Negative

${ }^{6}$ http://www.idmb.tamu.edu/gtl/ 
controls consisted of an MBP-tag to replace MBP-GiACS proteins for background subtraction. After the reaction, samples were subjected to a heptane extraction procedure to remove free palmitic acid, and the radioactivity of ${ }^{3} \mathrm{H}$-palmitoyl-CoA in the aqueous phase was counted in a Beckman Coulter LS 6000SE counter as described (Fritzler and Zhu, 2007; Fritzler et al., 2007). We also evaluated the effects of an ACS inhibitor, triacsin C (2,4,7-undecatrienal nitrosohydrazone; CAS 76896-80-5) on the GiACS activity, in which 1-32 $\mu \mathrm{M}$ of triacsin $\mathrm{C}$ was used to determine the $\mathrm{IC}_{50}$ value. In each experiment, there was a set of reactions under the same conditions, but without enzyme for use as controls for background subtractions.

\section{Efficacy of Triacsin C on the Growth of G. intestinalis In Vitro}

The effect of triacsin C on the growth of G. intestinalis (WB strain) in vitro was assessed by subculture in liquid medium as described (Arguello-Garcia et al., 2004). In this assay, $1 \times 10^{6}$ Giardia trophozoites were cultured in 4.5-mL screw-capped vials containing fresh TYI-S-33 medium (less than 1 week old) containing varied concentrations of Triacsin $\mathrm{C}(0.13-16 \mu \mathrm{M})$ for $24 \mathrm{~h}$ at $37^{\circ} \mathrm{C}$. Then $1 \times 10^{5}$ drug-exposed trophozoites were transferred to new $4.5-\mathrm{mL}$ vials containing drug-free medium and incubated for additional $48 \mathrm{~h}$ at $37^{\circ} \mathrm{C}$. Parasites were then counted and the parasite growth was expressed as the percent of surviving trophozoites in comparison to those in the negative controls that did not received an inhibitor.

\section{Results}

\section{The Genome of G. intestinalis Encodes Five Long Chain FA-CoA Synthetases that are Differentially Expressed the Parasite}

By data-mining the genome sequences of G. intestinalis (WB), we identified eight candidate genes encoding proteins that either exhibited high degree of identities with other known ACS proteins or annotated as long-chain FA CoA ligases or ACSs by the GiardiaDB (Table 1). Among them, five genes (designated as GiACS1 to GiACS5) appear to encode for ACS enzymes based on their identities to other ACS proteins and by the presence of AMP-binding domain, ACS signature motifs, and other active sites in their protein sequences (Figure 1C; Supplementary Figure S1). The top hit at the NCBI CDD for all five GiACS proteins is LC_FACS_euk (CDD No. cd05927) with expectation values (E-values) ranging from 1.92E-157 to 5.37E-117 (Table 1). Among the other three genes, GL50803_17170 (GenBank: XP_001707853) was annotated as "LCFA CoA ligase 5", and several short regions within the sequence could be mapped to the "VL_LC_FACS_like" domain at CDD (cd05907) with a much less significant E-value at 6.85E-19 (Table 1). The sequence also lacked most of the active sites in ACS, but only contained limited conserved amino acids at the two signature motifs, suggesting that GL50803_17170 was derived from an ancient very-long LC-FACS, but probably lost its ACS function. The remaining two genes were annotated as ACSs (GL50803_86511, GenBank: XP_001710279; and GL50803_16667, GenBank: XP_001709605)

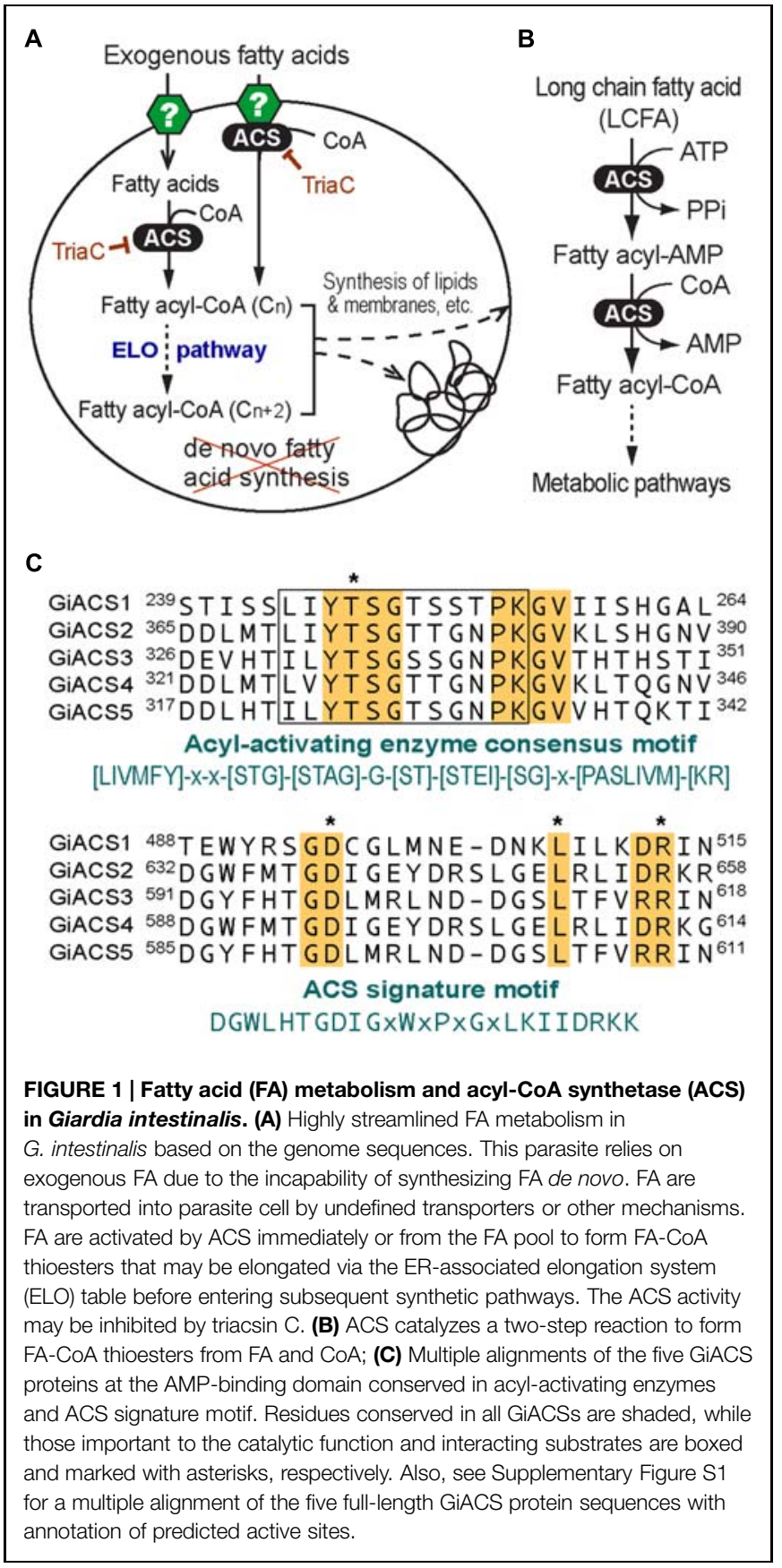

(Table 1). They both contained an ATP_grasp-5 domain (CDD: pfam549) with less significant $E$-values at $4.05 \mathrm{E}-38$ and $1.16 \mathrm{E}-$ 38 , respectively. Interestingly, proteins encoded by these two genes were ortholog of CoA-binding proteins from prokaryotes, suggesting they were derived from prokaryotic genes by lateral gene transfer and might more likely function as CoA-binding proteins than as ACS. Based on these observations, we concluded that the G. intestinalis genome encoded five ACSs and three ACS-related proteins.

Further transcriptome analysis by data-mining previously published transcriptome data generated using various platforms 
(i.e., SAGE, RNA-seq, and microarray) and available at GiardiaDB (Palm et al., 2005; Morf et al., 2010; Ringqvist et al., 2011; Franzen et al., 2013), we noticed that the five GiACS genes were differentially expressed in different parasite stages (Figure 2). In trophozoites, an earlier SAGE analysis detected the transcripts of four GiACS genes (with the exception of only GiACS4) (Figure 2A), whereas a more recent RNA-seq analysis was able to detect the transcripts of all five GiACS genes (Figure 2B). However, the general expression profiles in these two datasets were similar (i.e., the highest for GiACS3 and GiACS5, moderate for GiACS1 and GiACS2, and the lowest for GiACS4). In other stages including cysts and parasites during encystation and excystation, GiACS1 was the most highly expressed among all GiACS genes in all stages in the SAGE dataset (Figure 2A). GiACS1 also maintained the highest levels of expression during excystation process, but mid-level expressions during the encystation process (Figure 2A). GiACS2 was consistently expressed at moderate levels in all parasite stages, while GiACS3 had relatively high transcript levels in trophozoites and during encystation, but generally lower levels in cysts and excysation. GiACS4 was consistently the lowest or undetectable in all stages, whereas GiACS5 was highly expressed in trophozoites and during encystation, but slightly expressed during excystation (Figure 2A). In agreement with the SAGE analysis, a microarray analysis also detected apparent downregulation of GiACS3 and up-regulation of GiACS5 during the encystation process (Figure 2C). Although GiACS4 gene displays the lowest or even undetectable expression levels in all parasite life cycle stages, this gene significantly elevated its expression during the interaction with host cells or when culture medium is changed from DMEM to TYDK medium (Figure 2D). Although further functional studies are needed to delineate the biological roles played by the five Giardia ACS genes, their differential expressions in various biological processes and conditions clearly imply that they might play differential roles in the parasite life cycle.

\section{Insights from GiACS1 and GiACS2 Protein Models}

Considering the aforementioned data and the fact that chemotherapy in giardiasis is mostly directed against the trophozoite stage, the GiACS1, and GiACS2 proteins were
A

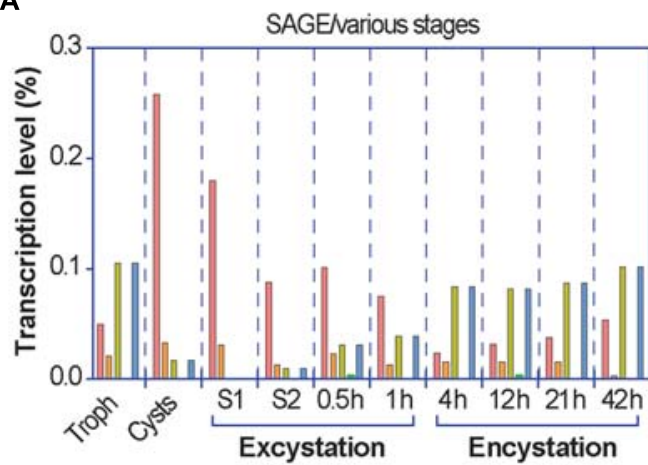

C

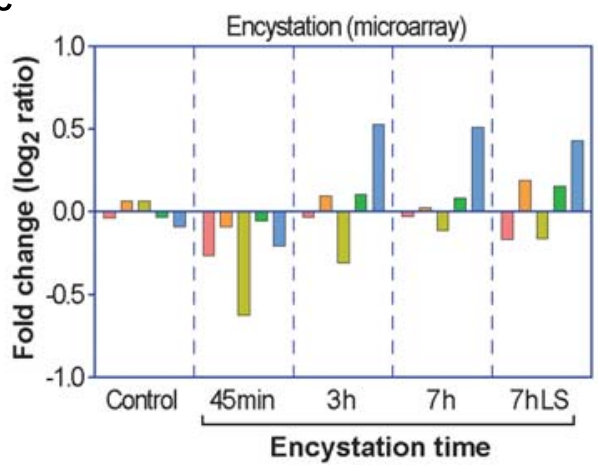

FIGURE 2 | Differential expressions of the five GiACS genes as determined by data-mining previously published transcriptome data available at GiardiaDB. All data were derived from $G$. intestinalis assemblage A WB strain. (A) Expression profiles based on serial analysis of gene expression (SAGE) in trophozoites (Troph), cysts, and during excystation and encystation (Palm et al., 2005). S1 (stage 1) = under acidic condition to mimic the stomach. S2 (stage 2) = trypsin and slight alkaline conditions to mimic the small intestines. (B) Transcriptional levels in trophozoites based on strand-specific RNA-seq using the Illumina HiSeq2000 platform (Franzen
B

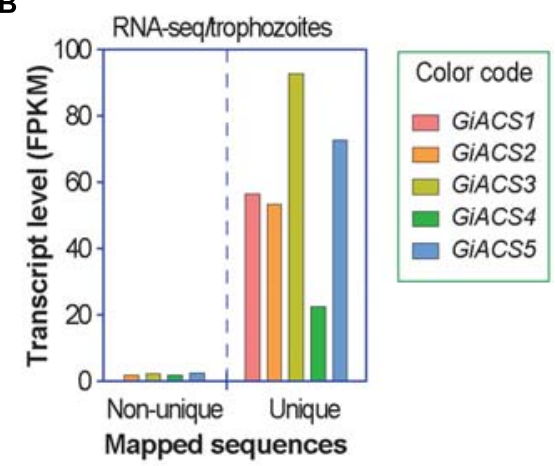

D

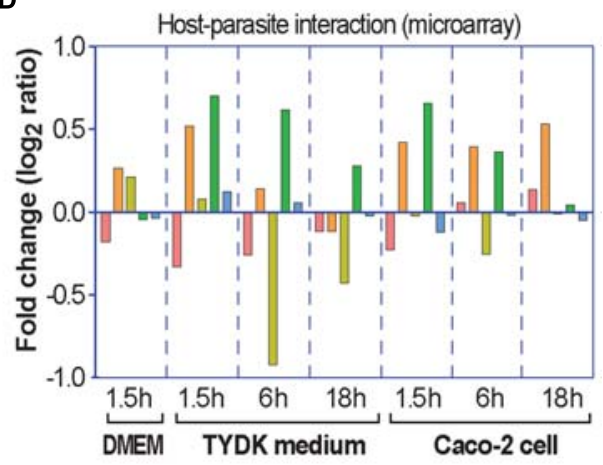

et al., 2013). FPKM = fragments per kilobase of exon model per million mapped reads; (C) Transcriptional changes in response to encystation stimuli based on a dual-color hybridization of a full-genome microarray analysis, in which encystation was induced in vitro for $45 \mathrm{~min}, 3 \mathrm{~h}$, and $7 \mathrm{~h}$ by standard 2-step protocol or for $7 \mathrm{~h}$ by lipid starvation (7hLS) (Morf et al., 2010). (D) Transcriptional changes in trophozoites during interacting with host cells as determined by a full-genome microarray analysis, in which trophozoites were used to infect Caco-2 cells in DMEM medium or cultured in cell-free TYDK medium for varied times (Ringqvist et al., 2011). 
considered as potential drug targets due to their stable and comparable transcription levels in trophozoites (Figure 2B), even though expression of both proteins differ upon trophozoiteepithelial cell interactions. GiACS2 was up regulated while GiACS1 was down regulated at $\leq 6 \mathrm{~h}$ of interacting with host cells (Figure 2D). In addition, GiACS1 is a much larger protein (853 aa) than the other GiACSs and their human counterparts (generally $<700$ aa); hence, the GiACS1 represents a divergent ACS.

In an initial mining of ACS crystals in $\mathrm{PDB}$, protozoan templates were absent. When the GiACS1 and GACS2 sequences were submitted to preliminary modeling, the retrieved models shared the highest scores with members of the CoA synthetase family as firefly luciferase, ACS of Saccharomyces cerevisiae and acetoacetyl-CoA synthetase of Streptomyces lividans (PDB IDs: 2D1S, 1RY2, and 4WD1, respectively). From these, the yeast homolog (1RY2) was chosen as template for refinement of protein models due to its ACS nature that closely matches with the proposed GiACS functions. In this way, a templatedirected modeling was performed and the two GiACS models (Figures 3A,D) had satisfactory scores: C-score: -2.57, TMscore $=0.636$, and RMSD $=0.95$ for GiACS1; C-score: -1.04 , TM-score $=0.773$, and RMSD $=1.65$ for GiACS2. These data reflect that GiACS models share 63.6\% (GiACS1) and $77.3 \%$ (GiACS2) homology (average deviation $<2 \AA$ ) with respect to the yeast ACS template. Also, the identity in sequence may be indicated with the available PDB structures resolved since I-Tasser is good for modeling protein targets in the "twilight zone" (20-30\% identity), which have no or weakly homologous templates (Roy et al., 2010).

In a further assessment of the quality for GiACS1/2 protein models, the Ramachandran plots were constructed. This tool

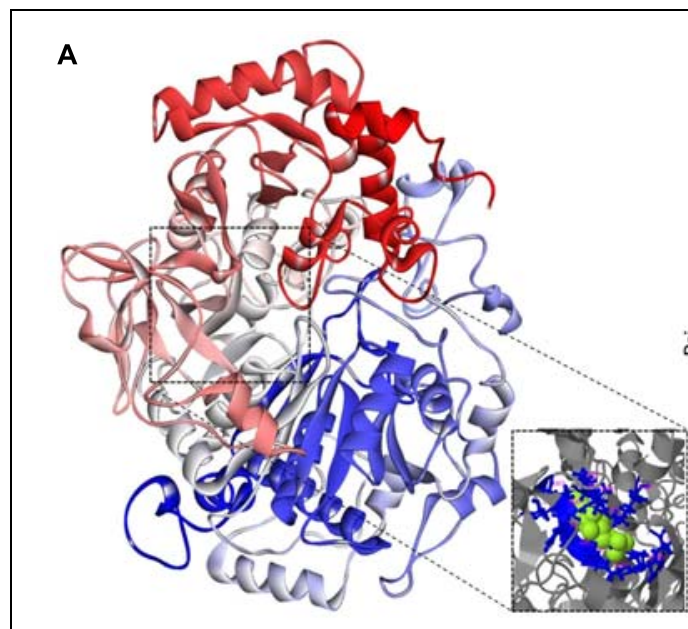

D

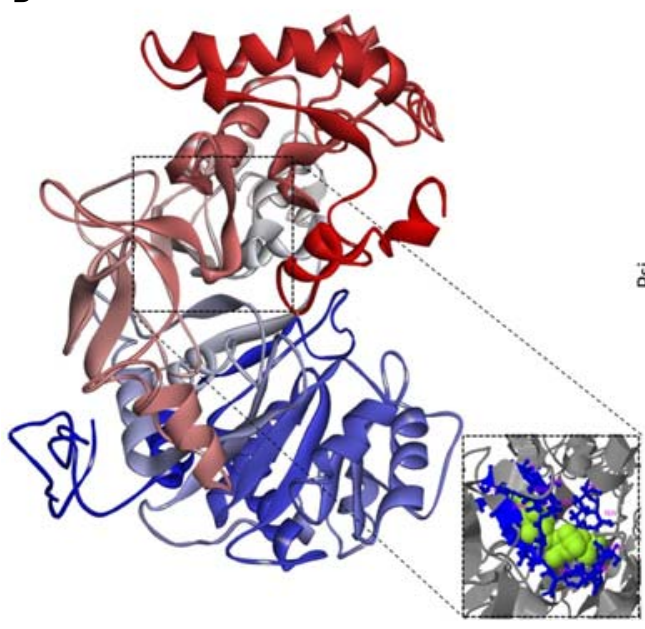

E

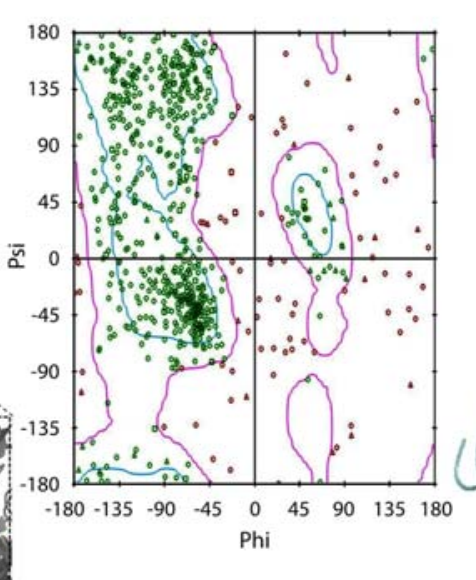

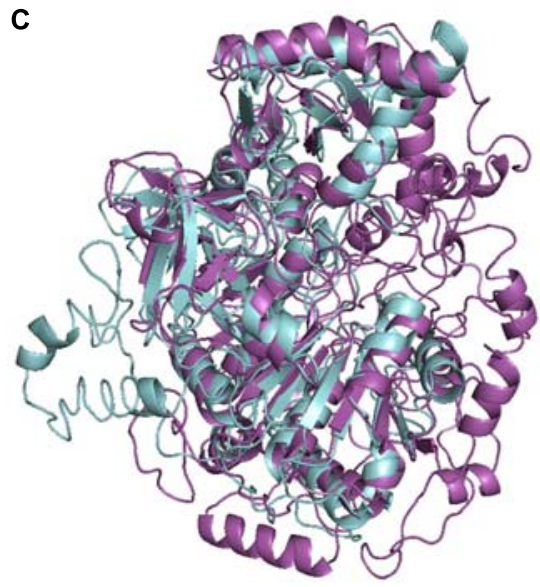

$\mathbf{F}$

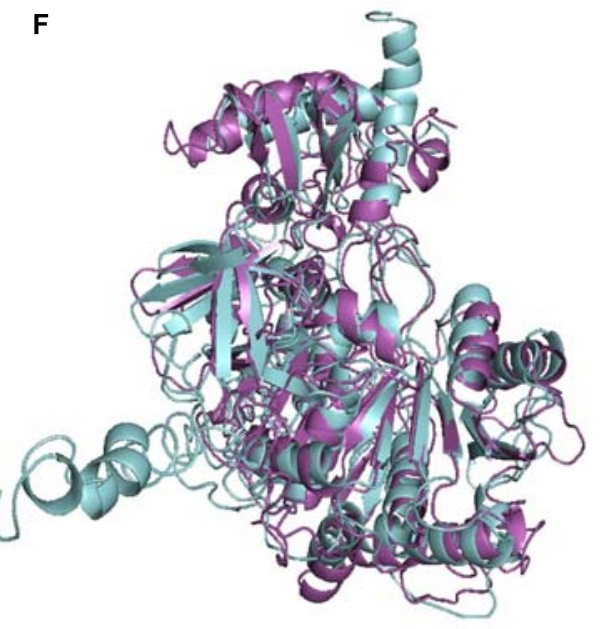

FIGURE 3 | Protein modeling features of GiACS1 and GiACS2. The protein folding models for GiACS1 (A) and GiACS2 (D) were retrieved using yeast ACS (PDB ID: 1RY2) as a template. Protein models are colored from $\mathrm{N}$-terminus (blue) to $\mathrm{C}$-terminus (red) and oriented in relation to the AMP/ATP heterogen (insets). The Ramachandran plots calculated for GiACS1 (B) and
GiACS2 (E) display glycine (triangles), proline (squares), and non-glycine non-proline (circles) residues. The structural alignments of GiACS1 (magenta) with HsACS5CRAc (cyan) (C) and 541 GiACS2 (magenta) with HsACS5b (cyan) (F) display folding differences between giardial and human counterparts. 
TABLE 3 | Ramachandran plots statistics of GiACS1 and GiACS2.

\begin{tabular}{lll}
\hline Residues in Ramachandran plot & $\begin{array}{l}\text { GiACS1 } \\
\text { (853 aa) }\end{array}$ & $\begin{array}{l}\text { GiACS2 } \\
\text { (693 aa) }\end{array}$ \\
\hline In most favored regions & $581(78.30 \%)$ & $443(72.63 \%)$ \\
In additionally allowed regions & $125(16.85 \%)$ & $104(17.04 \%)$ \\
In generously allowed or disallowed regions & $36(4.85 \%)$ & $63(10.33 \%)$ \\
Non-Gly and non-Pro residues & $742(100 \%)$ & $610(100 \%)$ \\
\# Gly (triangles) & 66 & 43 \\
\# Pro (squares) & 45 & 40 \\
\hline
\end{tabular}

not only predicts secondary structures from dihedral angles of individual amino acids ( $\varphi$ and $\psi$ ), but also provides distributions of residues in "favored regions" (contoured in blue line), "additionally allowed regions" (contoured in pink line), and the external "generously allowed" and "not allowed" regions as stated by the ProCheck platform (Figures 3B,E). The special cases of glycine and proline are ruled out as recommended and only residues contained in the two former regions were considered of satisfactory conformation. These distributions for GiACS1 and GiACS2 are listed in Table 3. In this, more than $75 \%$ of residues fall within favored regions, more than $15 \%$ fall within allowed regions and $<10 \%$ are in external regions. In summary, up to $95.15 \%$ of residues in GiACS1 and $89.67 \%$ of residues in GiACS2 have a satisfactory conformation within the protein structure predicted for these molecules; hence, the protein models obtained have an acceptable quality.

Further analyses to evaluate GiACS1 and GiACS2 as likely druggable targets were performed to determine the structural alignment of GiACSs with their most resembling human counterparts. In these, two isoforms of the human long chain fatty acyl CoA synthetases had the closest similarity to these GiACSs: the 5CRAc isoform (HsACS5CRAc, Acc. No. gb| EAW49534.1, $663 \mathrm{aa})$ displaying the highest expectation value (8e-76) and $37 \%$ of sequence identity with GiACS1 over a $64.1 \%$ coverage and the 5b isoform (HsACS5b, Acc. No. NP_976313.1, $683 \mathrm{aa})$ that displayed the highest expectation value $(5 \mathrm{e}-60)$ and $26 \%$ of sequence identity with GiACS2 over a $79.4 \%$ coverage. Despite the high $E$-values, the structural comparison of giardial and human counterparts revealed striking differences: GiACS1 and HsACS5CRAc had 31.0\% of structure identity over a span of 561 aa (RMSD: 3.20; TM-score: 0.769 normalized with HsACS5CRAc) while GiACS2 and HsACS5b shared 23.9\% of structure identity over a span of 594 aa (RMSD: 3.90; TMscore: 0.775 normalized with HsACS5b). In general, there were obvious folding differences between the Giardia and human ACS counterparts (Figures 3C,F), particularly in the case of GiACS1 as a consequence of the multiple insertions contained in this unusually large (853 aa) molecule. Based on these observations, it was tempting to compare purified GiACS in terms of catalytic abilities and susceptibility to specific inhibitors.

\section{Functional Confirmation of GiACS1 and GiACS2 as a Long Chain FA-CoA Synthetase}

After we determined that G. intestinalis possessed five ACSs, we decided to first investigate the biochemical features for two of them. We selected GiACS1 and GiACS2 in our initial study because both genes were relatively highly and consistently expressed in different life cycle stages, and GiACS2 appeared to be important in host-parasite interaction. Their whole ORFs were successfully cloned into the pMAL-c2E expression vector, and their products were expressed as MBP-fusion proteins (Figure 4A, inset). GiACS1 protein was purified into high purity, while the majority of GiACS2 was expressed at expected size, but some lower bands were present, suggesting some incomplete translation of GiACS2 probably due to the differences in codon usage between $G$. intestinalis and E. coli.

Using DTNB assay, we were able to individually evaluate the activity of GiACS1 and GiACS2 toward various saturated FA with chain lengths ranging from $\mathrm{C} 2: 0$ to C30:0. Both GiACS1 and GiACS2 displayed the highest activity over palmitic acid (C16:0) and myristic acid (C14:0) (Figure 4A). Their activity on other FA was much lower, mostly ranging from $\sim 5 \%$ to $\sim 25 \%$. These observations confirmed that both GiACS1 and GiACS2 are long-chain FACS with a relatively restricted substrate preference toward C14:0 and C16:0 FA. This feature makes these two GiACS proteins differ from the two Cryptosporidium ACSs that could use C12:0-C18:0 FA with comparable levels of activity (Guo et al., 2014). In kinetic studies, these two enzymes exhibited allosteric and Michaelis-Menten kinetics toward palmitic acid and ATP, respectively (Figures $\mathbf{4 B}, \mathbf{C}$ ). Their kinetics parameters are listed in Table 4. We also validated the function of GiACS1 and GiACS2 by directly detecting the formation of palmitoylCoA using a radioactive assay, in which both enzymes exhibited specific activities comparable to those obtained using DTNB assay (Figure 4E).

\section{Triacsin C Inhibits GiACS1 and GiACS2 Enzyme Activity as Well as the In Vitro Growth of G. intestinalis}

We further tested whether GiACS1 and GiACS2 were amendable to the inhibition by an ACS inhibitor triacsin C, and observed

TABLE 4 | Kinetic parameters for GiACS1 and GiACS2 ${ }^{\text {. }}$

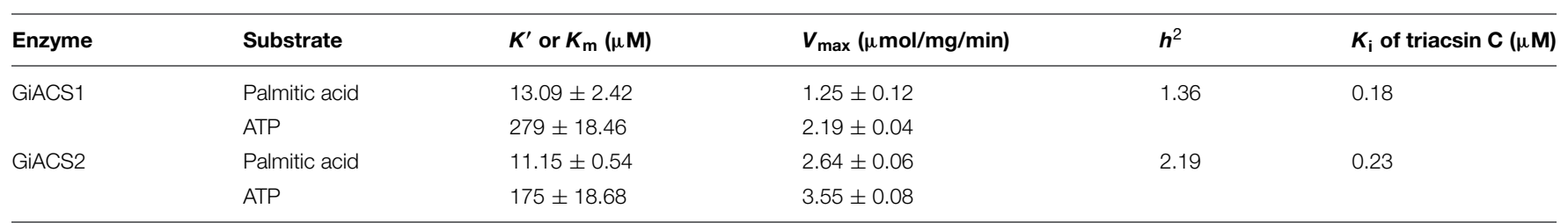

${ }^{1}$ Values for $K^{\prime}, K_{\mathrm{m}}$, and $V_{\max }$ are expressed as mean $\pm S D ;{ }^{2} h=$ Hill coefficient. 

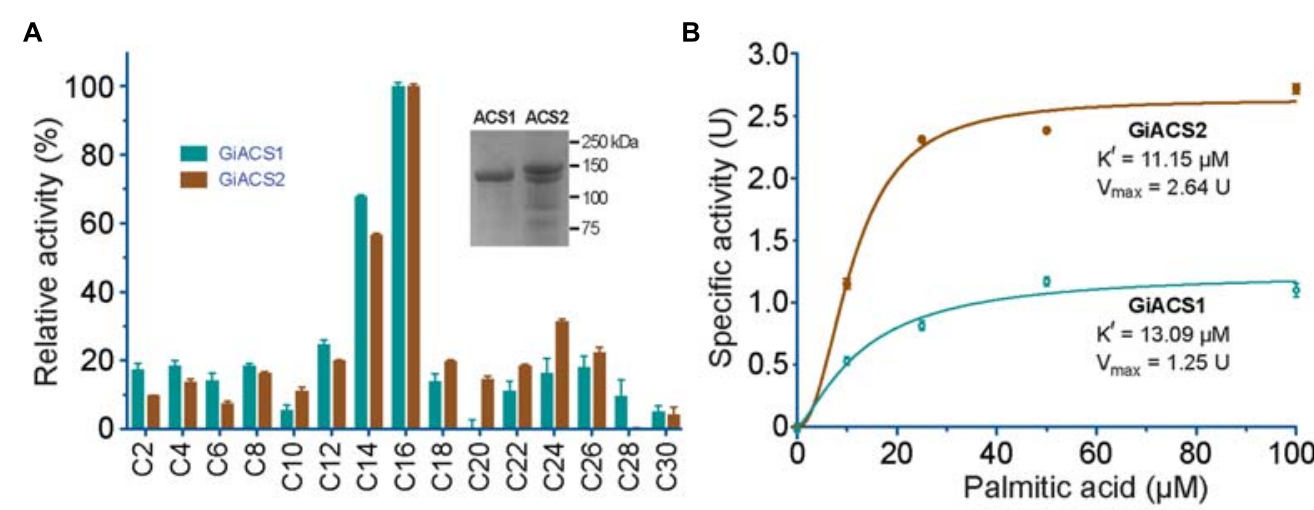

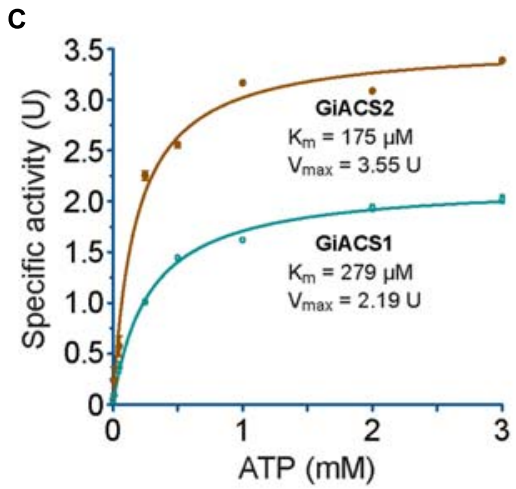

FIGURE 4 | Enzyme kinetic features of recombinant GiACS1 and GiACS2 as determined by a 5, 5'-dithio-bis-(2-nitrobenzoate) (DTNB) colorimetric assay. (A) Substrate preference of GiACS1 and GiACS2 toward FA with varied carbon chain lengths. Relative activities were shown in comparison with that on the C16:0 palmitic acid. Inset showed SDS-PAGE analysis of purified recombinant GiACS1 and GiACS2 as maltose-binding protein (MBP)-fusion proteins; (B) Allosteric kinetics of
D

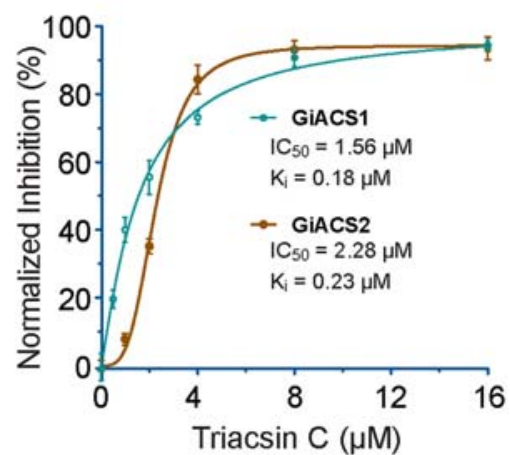

E

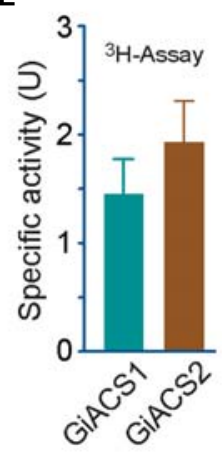

GiACS proteins on palmitic acid; (C) Michaelis-Menten kinetics of GiACS on ATP; (D) Inhibition of triacsin $\mathrm{C}$ on the GiACS enzyme activity; and (E) Enzyme activity by detecting the formation of ${ }^{3} \mathrm{H}$-palmitoyl-CoA using a heptane extraction-based radioactive assay. Bars represent SEM derived from three or more reactions. At least two independent assays were performed for each experiment, and the data from one representative assay were shown here. $U=\mu \mathrm{mol} / \mathrm{mg} / \mathrm{min}$. that this compound could inhibit their enzyme activity with IC $_{50}$ values at 1.56 and $2.28 \mu \mathrm{M}$ on GiACS1 and GiACS2, respectively (Figure 4D). Their corresponding $\boldsymbol{K}_{\mathrm{i}}$ values were at 0.18 and $0.23 \mu \mathrm{M}$, respectively, based on a competitive inhibition model (Cheng and Prusoff, 1973) (Figure 4D). The efficacy data were comparable to those observed for the two ACSs from the apicomplexan parasite Cryptosporidium parvum (i.e., $\mathbf{I C}_{50}$ values at 3.70 and $2.32 \mu \mathrm{M}$ for CpACS1 and CpACS2, respectively) (Guo et al., 2014).

Since the major goal of this study is to explore the potential of GiACSs to serve as drug targets, we further investigated the in vitro efficacy of triacsin $C$ against the growth of $G$. intestinalis in axenic culture. We observed that triacsin $\mathrm{C}$ indeed effectively inhibited the parasite growth in a dose-dependent manner with an estimated $\mathrm{IC}_{50}$ value at $0.8 \mu \mathrm{M}$ (Figure 5). At other tested doses, $2.2 \mu \mathrm{M}$ and $\geq 10 \mu \mathrm{M}$ triacsin $\mathrm{C}$ achieved 90 and $100 \%$ inhibitions, respectively.

\section{Discussion}

FA are essential to all organisms as one of the major components of biomembranes. In most organisms, FA also serves as an

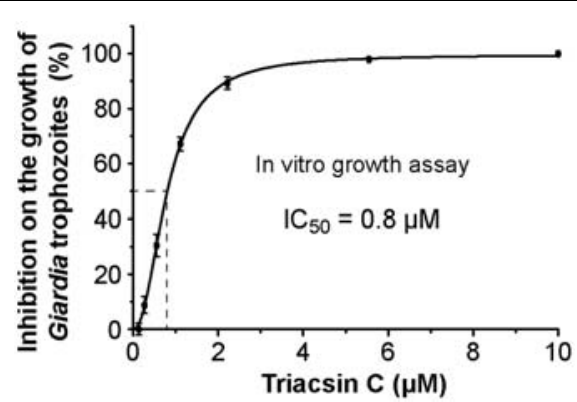

FIGURE 5 | Efficacy of triacsin C on the growth of G. intestinalis (WB strain) in vitro, in which axenically cultured trophozoites were treated with various doses of inhibitor for $\mathbf{2 4} \mathrm{h}$, transferred to drug-free medium, and allowed to grow for additional $48 \mathrm{~h}$ before being counted for calculating the parasite growth.

energy source. Because FA needs to be activated to form FA-CoA before they can enter subsequent metabolic pathways, enzymes catalyzing the formation of FA-CoA are essential and considered as potential drug targets. We have recently reported that ACS enzymes could serve as effective drug targets in Cryptosporidium 
(Guo et al., 2014), which prompted us to explore whether ACSs could also be targeted for developing novel anti-Giardia drugs. Indeed, in the present study, we have confirmed that the ACS inhibitor could inhibit not only the reactions catalyzed by GiACS2, but also the in vitro growth of G. intestinalis at submicromolar levels (Figure 5). The possibility to consider GiACS1 and GiACS2 as likely drug targets was further supported by bioinformatics data in which protein modeling analyses showed structural differences between giardial and human counterparts (Figure 3F). This fact could be the result of evolutionary processes in which the giardial ACSs are likely ancestors of other ACSs counterparts. In this context, it is conceivable that GiACS1 (853 aa) could have had a reductive process during evolution. In spite of the predicted satisfactory quality of the protein models in this study, further crystallographic studies in purified GiACS1/2 will offer additional insights not only for site-targeted drug design, but also to assess if a differential adaptation in the catalytic pocket of GiACS2, as compared to GiACS1, exists. Moreover, the possibility to recover enzyme activities from recombinant GiACS1/2 will allow refining crystallographic analyses at distinct enzyme conformations and under interaction with Triacsin $\mathrm{C}$ or other specific inhibitors. This renders an advantage over the failure to detect enzyme activities in other parasitic recombinant ACSs (Matesanz et al., 1999; Guo et al., 2014).

Triacsin C is an analog of polyunsaturated FA that was first isolated from the fungal Streptomyces aureofaciens (Omura et al., 1986). It is known as a long-chain ACS-specific inhibitor with little effect on short-chain or mitochondrial-type ACSs in mammals (Omura et al., 1986; Tomoda et al., 1987; Hartman et al., 1989; Van Horn et al., 2005). Mammals possess six genes encoding long-chain ACS enzymes that are designated as ACSL1-6 including some variants produced by alternative intronsplicing with varied spectra of substrate preferences (Soupene and Kuypers, 2008; Watkins and Ellis, 2012). Among them, triacsin

\section{References}

Ali, V., and Nozaki, T. (2007). Current therapeutics, their problems, and sulfurcontaining-amino-acid metabolism as a novel target against infections by "amitochondriate" protozoan parasites. Clin. Microbiol. Rev. 20, 164-187. doi: 10.1128/CMR.00019-06

Arguello-Garcia, R., Cruz-Soto, M., Romero-Montoya, L., and Ortega-Pierres, G. (2004). Variability and variation in drug susceptibility among Giardia duodenalis isolates and clones exposed to 5-nitroimidazoles and benzimidazoles in vitro. J. Antimicrob. Chemother. 54, 711-721. doi: 10.1093/jac/ dkh388

Beach, D. H., Holz, G. G. Jr., Singh, B. N., and Lindmark, D. G. (1990). Fatty acid and sterol metabolism of cultured Trichomonas vaginalis and Tritrichomonas foetus. Mol. Biochem. Parasitol. 38, 175-190. doi: 10.1016/0166-6851(90) 90021-D

Bernson, V. S. M. (1976). Acetyl-CoA hydrolase; activity, regulation and physiological significance of the enzyme in brown adipose tissue from hamster. Eur. J. Biochem. 67, 403-410. doi: 10.1111/j.1432-1033.1976.tb1 0705.x

Busatti, H. G., Santos, J. F., and Gomes, M. A. (2009). The old and new therapeutic approaches to the treatment of giardiasis: where are we? Biologics 3, 273-287.

Cheng, Y., and Prusoff, W. H. (1973). Relationship between the inhibition constant (K1) and the concentration of inhibitor which causes 50 per cent inhibition (I50) of an enzymatic reaction. Biochem. Pharmacol. 22, 3099-3108. doi: 10.1016/0006-2952(73)90196-2
$\mathrm{C}$ is an effective inhibitor for ACSL1, ACSL3, and ACSL4 (IC 50 values between 4 and $7.5 \mu \mathrm{M}$ ), but not for ACSL5, ACS6_v1, and ACS6_v2 (Van Horn et al., 2005). The abundance of ACSLs and their varied sensitivities to triacsin $\mathrm{C}$ might explain its ineffectiveness on the recycling of FA into phospholipids in mammalian cells (Igal et al., 1997) and its selective inhibition on Cryptosporidium both in vitro and in vivo (Guo et al., 2014). Collectively, the present study not only supports the notion that ACS enzymes can be explored as drug targets in Giardia, but also provides strong proof-of-concept data for the further identification of triacsin $\mathrm{C}$ analogs and other classes of small molecules for developing more selective inhibitors against the parasite.

\section{Acknowledgments}

This research was supported in part by grants funded by the Texas A\&M University-CONACYT Collaborative Research Program (Proposal No. 2010-012 to GZ and GO-P), and by the National Institute of Allergy and Infectious Diseases, National Institutes of Health (grant R21 AI119710 and R01 AI44594 to GZ). We also thank GiardiaDB (http://www.giardiadb.org/) and investigators who made their genome and transcriptome data available at GiardiaDB. We are grateful to Arturo Pérez Taylor for informatics assistance and Alejandro Sosa for his useful comments on protein modeling.

\section{Supplementary Material}

The Supplementary Material for this article can be found online at: http://journal.frontiersin.org/article/10.3389/fmicb. 2015.00753

Das, S., Stevens, T., Castillo, C., Villasenor, A., Arredondo, H., and Reddy, K. (2002). Lipid metabolism in mucous-dwelling amitochondriate protozoa. Int. J. Parasitol. 32, 655-675. doi: 10.1016/S0020-7519(02)00006-1

Feng, Y., and Xiao, L. (2011). Zoonotic potential and molecular epidemiology of Giardia species and giardiasis. Clin. Microbiol. Rev. 24, 110-140. doi: 10.1128/CMR.00033-10

Franzen, O., Jerlstrom-Hultqvist, J., Einarsson, E., Ankarklev, J., Ferella, M., Andersson, B., et al. (2013). Transcriptome profiling of Giardia intestinalis using strand-specific RNA-seq. PLoS Comput. Biol. 9:e1003000. doi: 10.1371/journal.pcbi.1003000

Fritzler, J. M., Millership, J. J., and Zhu, G. (2007). Cryptosporidium parvum long-chain fatty acid elongase. Eukaryot. Cell 6, 2018-2028. doi: 10.1128/ec.00 210-07

Fritzler, J. M., and Zhu, G. (2007). Functional characterization of the acyl-[acyl carrier protein] ligase in the Cryptosporidium parvum giant polyketide synthase. Int. J. Parasitol. 37, 307-316. doi: 10.1016/j.ijpara.2006.10.014

Granados, C. E., Reveiz, L., Uribe, L. G., and Criollo, C. P. (2012). Drugs for treating giardiasis. Cochrane Database. Syst. Rev. 12, CD007787. doi: 10.1002/14651858.CD007787.pub2

Guo, F., Zhang, H., Fritzler, J. M., Rider, S. D. Jr., Xiang, L., McNair, N. N., et al. (2014). Amelioration of Cryptosporidium parvum infection in vitro and in vivo by targeting parasite fatty acyl-coenzyme A synthetases. J. Infect. Dis. 209, 1279-1287. doi: 10.1093/infdis/jit645

Guo, F., and Zhu, G. (2012). Presence and removal of a contaminating $\mathrm{NADH}$ oxidation activity in recombinant maltose-binding protein fusion 
proteins expressed in Escherichia coli. Biotechniques 52, 247-253. doi: $10.2144 / 0000113822$

Hartman, E. J., Omura, S., and Laposata, M. (1989). Triacsin C: a differential inhibitor of arachidonoyl-CoA synthetase and nonspecific long chain acyl-CoA synthetase. Prostaglandins 37, 655-671. doi: 10.1016/0090-6980(89)90103-2

Hlavsa, M. C., Watson, J. C., and Beach, M. J. (2005). Giardiasis surveillanceUnited States, 1998-2002. MMWR Surveill. Summ. 54, 9-16.

Igal, R. A., Wang, P., and Coleman, R. A. (1997). Triacsin C blocks de novo synthesis of glycerolipids and cholesterol esters but not recycling of fatty acid into phospholipid: evidence for functionally separate pools of acyl-CoA. Biochem. J. 324 (Pt 2), 529-534.

Jarroll, E. L., Muller, P. J., Meyer, E. A., and Morse, S. A. (1981). Lipid and carbohydrate metabolism of Giardia lamblia. Mol. Biochem. Parasitol. 2, 187-196. doi: 10.1016/0166-6851(81)90099-2

Lalle, M. (2010). Giardiasis in the post genomic era: treatment, drug resistance and novel therapeutic perspectives. Infect. Disord. Drug Targets 10, 283-294. doi: $10.2174 / 187152610791591610$

Lee, S. H., Stephens, J. L., and Englund, P. T. (2007). A fatty-acid synthesis mechanism specialized for parasitism. Nat. Rev. Microbiol. 5, 287-297. doi: 10.1038/nrmicro1617

Matesanz, F., Duran-Chica, I., and Alcina, A. (1999). The cloning and expression of Pfacs1, a Plasmodium falciparum fatty acyl coenzyme A synthetase-1 targeted to the host erythrocyte cytoplasm. J. Mol. Biol. 291, 59-70. doi: 10.1006/jmbi.1999.2964

Morf, L., Spycher, C., Rehrauer, H., Fournier, C. A., Morrison, H. G., and Hehl, A. B. (2010). The transcriptional response to encystation stimuli in Giardia lamblia is restricted to a small set of genes. Eukaryot. Cell 9, 1566-1576. doi: 10.1128/EC.00100-10

Morrison, H. G., Mcarthur, A. G., Gillin, F. D., Aley, S. B., Adam, R. D., Olsen, G. J., et al. (2007). Genomic minimalism in the early diverging intestinal parasite Giardia lamblia. Science 317, 1921-1926. doi: 10.1126/science.1143837

Omura, S., Tomoda, H., Xu, Q. M., Takahashi, Y., and Iwai, Y. (1986). Triacsins, new inhibitors of acyl-CoA synthetase produced by Streptomyces sp. J. Antibiot. 39, 1211-1218. doi: 10.7164/antibiotics.39.1211

Palm, D., Weiland, M., Mcarthur, A. G., Winiecka-Krusnell, J., Cipriano, M. J., Birkeland, S. R., et al. (2005). Developmental changes in the adhesive disk during Giardia differentiation. Mol. Biochem. Parasitol. 141, 199-207. doi: 10.1016/j.molbiopara.2005.03.005

Plutzer, J., Ongerth, J., and Karanis, P. (2010). Giardia taxonomy, phylogeny and epidemiology: facts and open questions. Int. J. Hyg. Environ. Health 213, 321-333. doi: 10.1016/j.ijheh.2010.06.005

Ringqvist, E., Avesson, L., Soderbom, F., and Svard, S. G. (2011). Transcriptional changes in Giardia during host-parasite interactions. Int. J. Parasitol. 41, 277-285. doi: 10.1016/j.ijpara.2010.09.011

Roy, A., Kucukural, A., and Zhang, Y. (2010). I-TASSER: a unified platform for automated protein structure and function prediction. Nat. Protoc. 5, 725-738. doi: $10.1038 /$ nprot.2010.5

Soupene, E., and Kuypers, F. A. (2008). Mammalian long-chain acyl-CoA synthetases. Exp. Biol. Med. 233, 507-521. doi: 10.3181/0710-MR-287
Tejman-Yarden, N., and Eckmann, L. (2011). New approaches to the treatment of giardiasis. Curr. Opin. Infect. Dis. 24, 451-456. doi: 10.1097/QCO.0b013e32834ad401

Tian, H. F., Chen, B., and Wen, J. F. (2010). Giardiasis, drug resistance, and new target discovery. Infect. Disord. Drug Targets 10, 295-302. doi: $10.2174 / 187152610791591629$

Tomoda, H., Igarashi, K., and Omura, S. (1987). Inhibition of acyl-CoA synthetase by triacsins. Biochim. Biophys. Acta 921, 595-598. doi: 10.1016/00052760(87)90088-9

Van Horn, C. G., Caviglia, J. M., Li, L. O., Wang, S., Granger, D. A., and Coleman, R. A. (2005). Characterization of recombinant long-chain rat acylCoA synthetase isoforms 3 and 6: identification of a novel variant of isoform 6 . Biochemistry 44, 1635-1642. doi: 10.1021/bi0477211

Watkins, P. A., and Ellis, J. M. (2012). Peroxisomal acyl-CoA synthetases. Biochim. Biophys. Acta 1822, 1411-1420. doi: 10.1016/j.bbadis.2012.02.010

Yang, J., Yan, R., Roy, A., Xu, D., Poisson, J., and Zhang, Y. (2015). The I-TASSER Suite: protein structure and function prediction. Nat. Methods 12, 7-8. doi: 10.1038/nmeth.3213

Yoder, J. S., Beach, M. J., and Centers for Disease Control and Prevention. (2007). Giardiasis surveillance-United States, 2003-2005. MMWR Surveill. Summ. 56, 11-18.

Yoder, J. S., Gargano, J. W., Wallace, R. M., Beach, M. J., and Centers for Disease Control and Prevention. (2012). Giardiasis surveillance-United States, 2009-2010. MMWR Surveill. Summ. 61, 13-23.

Yoder, J. S., Harral, C., Beach, M. J., and Centers for Disease Control and Prevention. (2010). Giardiasis surveillance - United States, 2006-2008. MMWR Surveill. Summ. 59, 15-25.

Yu, Y., Zhang, H., Guo, F., Sun, M., and Zhu, G. (2014). A unique hexokinase in Cryptosporidium parvum, an apicomplexan pathogen lacking the Krebs cycle and oxidative phosphorylation. Protist 165, 701-714. doi: 10.1016/j.protis.2014.08.002

Zhang, Y. (2008). I-TASSER server for protein 3D structure prediction. BMC Bioinformatics 9:40. doi: 10.1186/1471-2105-9-40

Zhuravleva, E., Gut, H., Hynx, D., Marcellin, D., Bleck, C. K., Genoud, C., et al. (2012). Acyl coenzyme A thioesterase Them5/Acot15 is involved in cardiolipin remodeling and fatty liver development. Mol. Cell. Biol. 32, 2685-2697. doi: 10.1128/MCB.00312-12

Conflict of Interest Statement: The authors declare that the research was conducted in the absence of any commercial or financial relationships that could be construed as a potential conflict of interest.

Copyright (c) 2015 Guo, Ortega-Pierres, Argüello-García, Zhang and Zhu. This is an open-access article distributed under the terms of the Creative Commons Attribution License (CC BY). The use, distribution or reproduction in other forums is permitted, provided the original author(s) or licensor are credited and that the original publication in this journal is cited, in accordance with accepted academic practice. No use, distribution or reproduction is permitted which does not comply with these terms. 